\title{
Heavy-light mesons with domain wall fermions
}

\section{Shigemi Ohta*}

Inst. Particle and Nuclear Studies, KEK and Physics Department, Sokendai, Tsukuba, Ibaraki 305-0801, Japan and RIKEN BNL Research Center, BNL, Upton, NY 11973, USA

E-mail: shigemi.ohta@kek.jp

\section{HueyWen Lin}

Physics Department, Columbia University, New York, NY 10027, USA

E-mail: hwlinephysics.columbia.edu

\section{Norikazu Yamada}

Inst. Particle and Nuclear Studies, KEK and Physics Department, Sokendai, Tsukuba, Ibaraki 305-0801, Japan

E-mail: norikazu.yamada@kek.jp

\section{[RBC Collaboration]}

We present RBC heavy-light meson spectroscopy with quenched DBW2 gauge configurations at lattice cutoff $a^{-1}$ of about $3 \mathrm{GeV}$. Both heavy and light quarks are described by domain-wall fermions (DWF). The heavy quark mass ranges between 0.1 and 0.4 lattice units, covering charm. The light quark mass ranges between 0.008 and 0.04 , covering strange. In particular, we discuss charmed $\left(D\right.$ and $\left.D^{*}\right)$ and charm-strange $\left(D_{s}\right.$ and $\left.D_{s J}\right)$ mesons with spin-parity $J^{P}=0^{ \pm}$and $1^{ \pm}$. The preliminary results indicate that DWF describe charm on the quenched DBW2 ensemble at this cutoff. The masses of the $J^{P}=0^{ \pm}$and $1^{ \pm} D, D^{*}, D_{s}$ and $D_{S J}$ meson states are well reproduced to within a few $\%$; their parity splitting, $\Delta_{J}$, are better reproduced than previous works, with only 10-20\% over estimations; the experimental observation that the splitting for non-strange states, $\Delta_{u d}$, is bigger than that for strange states, $\Delta_{s}$, is reproduced as well; but the hyperfine splittings are only $60-65 \%$ reproduced. Regarding the depenence on heavy quark mass, $\Delta_{J=0}$ and $\Delta_{J=1}$ are degenerate for $m_{\text {heavy }} a>0.2-0.3 ; \Delta_{J=0}$ increases as $m_{\text {heavy }}$ decreases further while $\Delta_{J=1}$ does not.

XXIIIrd International Symposium on Lattice Field Theory

25-30 July 2005

Trinity College, Dublin, Ireland

\footnotetext{
*Speaker.
} 


\section{Introduction}

We report preliminary results from a RIKEN-BNL-Columbia (RBC) lattice numerical calculation on charmed meson spectroscopy with domain-wall fermions (DWF) [1] quarks and a quenched gauge ensemble with the rectangular improved DBW2 (Doubly-Blocked Wilson 2) [2] action at the lattice cutoff $a^{-1}$ of about $3 \mathrm{GeV}$. All the quarks including charm are described by DWF.

There are two main motivations: 1) By implementing charm as DWF, difficulties arising from lacking the Glashow-Illiopoulos-Miani mechanism can be avoided, there by making "charm-in" lattice calculations of hadron electroweak transition easier. This calculation serves as a test for such projects. 2) Recent discoveries of $D_{S J}$ mesons by the B factory experiments made comparison of lattice calculations and experiments more interesting. Planned experiments at $\tau$-charm factories will make this more accurate and challenging.

In Table 1 we summarize low-lying chamed $\left(D\right.$ and $\left.D^{*}\right)$ and charm-strange $\left(D_{s}\right.$ and $\left.D_{S J}\right)$ mesons mass: spin-pariity $J^{P}=0^{\mp}$ and $1^{\mp}$ states are known. From these we identify the following objectives for the present calculation to explain: The splitting between parity partners, $\Delta_{J}=m_{D_{l}}\left(J^{+}\right)-m_{D_{l}}\left(J^{-}\right)$(see Table 2.) A) They appear insensitive to the spin $J$,

$$
\Delta_{l, J=0} \sim \Delta_{l, J=1} .
$$

Here the subscript $l$ refers to the light quark flavor, either non-strange $(u d)$ or strange $(s)$. B) On the other hand they seem dependent on $m_{l}$,

$$
\Delta_{u d}>\Delta_{s}
$$

C) The hyperfine splitting $\Delta_{\mathrm{hf}}=m_{D_{l}}\left(1^{-}\right)-m_{D_{l}}\left(0^{-}\right)$or $m_{D_{l}}\left(1^{+}\right)-m_{D_{l}}\left(0^{+}\right)$(see Table 3.) They seem independent of $m_{l}$ or parity.

Before the discoveries of $D_{S J}$ mesons, Bardeen, Eichten and Hill [3], based on their $S U(3)_{L} \times$ $S U(3)_{R}$ model for heavy-light meson systems, made a set of predictions regarding the $D$ and $D_{s}$ spectroscopy: that the parity splitting $\Delta_{l J}$ would be independent of the spin $J$ and that it is weakly dependent on the heavy quark mass. They also made an interesting prediction that a GolebergerTreiman like relation should hold in the pion emission from excited $D^{*}$ or $D_{s J}$ states: $g_{\pi}=\Delta_{l J} / f_{\pi}$, though this is not in the scope of current study. Nowak, Rho and Zahed [4] made similar predictions based on a different model. More recently Becirevic, Fajfer and Prelovsek [5], based on yet another model, discussed it is difficult to understand the experimental observation that $\Delta_{u d}>\Delta_{s}$.

By now many lattice numerical studies $[6,7,8,9,10,11]$ have been conducted on these and related meson states. To summarize their results: a) The majority $[6,7,8,9]$ have overestimated

\begin{tabular}{ll}
\multicolumn{1}{c}{$J^{P}$} & \multicolumn{1}{c}{ mass $(\mathrm{MeV})$} \\
\hline \hline$D^{ \pm}\left(0^{-}\right)$ & $1869.4(5)$ \\
$D^{* \pm}\left(1^{-}\right)$ & $2010.0(5)$ \\
$D_{0}^{*}\left(0^{+} ?\right)$ & $2308(17)(15)(28)$ \\
$D_{1}^{\prime}\left(1^{+}\right)$ & $2427(26)(20)(15)$
\end{tabular}

\begin{tabular}{ll}
\multicolumn{1}{c}{$J^{P}$} & mass $(\mathrm{MeV})$ \\
\hline \hline$D_{s}^{ \pm}\left(0^{-}\right)$ & $1968.3(5)$ \\
$D_{s}^{* \pm}\left(1^{-} ?\right)$ & $2112.1(7)$ \\
$D_{s 0}^{* \pm}\left(0^{+}\right)$ & $2317.4(9)$ \\
$D_{s 1}^{* \pm}\left(1^{+}\right)$ & $2459.3(1.3)$
\end{tabular}

Table 1: Experimental values of low-lying $D$ and $D^{*}$ (left) and $D_{s}$ and $D_{s J}$ (right) mass. 


\begin{tabular}{cll} 
light quark & $0^{+}-0^{-}(\mathrm{MeV})$ & $1^{+}-1^{-}(\mathrm{MeV})$ \\
\hline \hline$s$ & 349.1 & 347.2 \\
$u d$ & 439 & 417
\end{tabular}

Table 2: Mass splitting, $\Delta_{l J}$, between parity partners.

the parity splittings, $\Delta_{J}$, with values typically about $500 \mathrm{MeV}$. b) A quenched calculation underestimated the hyperfine splittings reproducing only about half of the experimental values [6]. This is considered a common pathology for quenched calculations. c) Some success was observed in reproducing the degeneracy of $\Delta_{J=0} \sim \Delta_{J=1}$ [11]. The majority of these lattice studies employ static or non-relativistic description of the heavy charm quark, with the exception of ref. [9] which employs the Fermilab method. In contrast, in this study we explore the possibility of propagating the charm quark as a domain-wall fermion on a high-cutoff lattice. Needless to say, we exploit the good chiral symmetry of DWF for the light quarks.

\section{Numerical Method}

The quenched DBW2 gauge ensemble we use in this study is described in a previous RBC publication [12]. 103 configurations of the total 106 described there are used here. The gauge coupling is set at $\beta=1.22$. The lattice volume is $24^{3} \times 48$ and corresponds to about $(1.6 \mathrm{fm})^{3}$ spatial box as the lattice cutoff measured by the $\rho$-meson mass is $2.914(54) \mathrm{GeV}$. We will use this cutoff estimate throughout this report. If we use the static quark potential instead, we obtain 3.07 $\mathrm{GeV}$ [13]. The difference gives an estimate of quenched systematics.

All the four quark flavors are described as domain-wall fermions (see, for example, ref. [14] for the details of numerical implementation.) We set the fifth-dimension lattice extent as $L_{S}=10$, the domain wall height $M_{5}=1.65$. The propagators are calculated with "periodic+antiperiodic" boundary condition in time, so the hadron propagators are periodic with a period of 96.

For the light quark mass, five bare values of 0.008, 0.016, 0.024, 0.032 and 0.040 are used. From the calculated light-light pseudoscalar mass, the strange quark mass is known to be $0.0295(14)$ in lattice units [12].

For the heavy quark mass, five values of $0.1,0.2,0.3,0.4$ and 0.5 are used. Here a possible problem is that the domain wall fermion with explicit mass of $O(1)$ may no longer be localized to the domain wall. Jun Noaki of the RBC Collaboration investigated this issue by looking at the dependence on the fifth coordinate, $s$, of the four-dimensional norms of the low-lying DWF eigenmodes. As is presented in Figure 1, the DWF are localized to the wall for explicit mass values smaller than about 0.5 .

\begin{tabular}{cll} 
light quark & $1^{-}-0^{-}(\mathrm{MeV})$ & $1^{+}-0^{+}(\mathrm{MeV})$ \\
\hline \hline$s$ & 143.8 & 141.9 \\
$u d$ & 140.6 & 119
\end{tabular}

Table 3: Hyperfine splitting $\Delta_{\mathrm{hf}}$ 


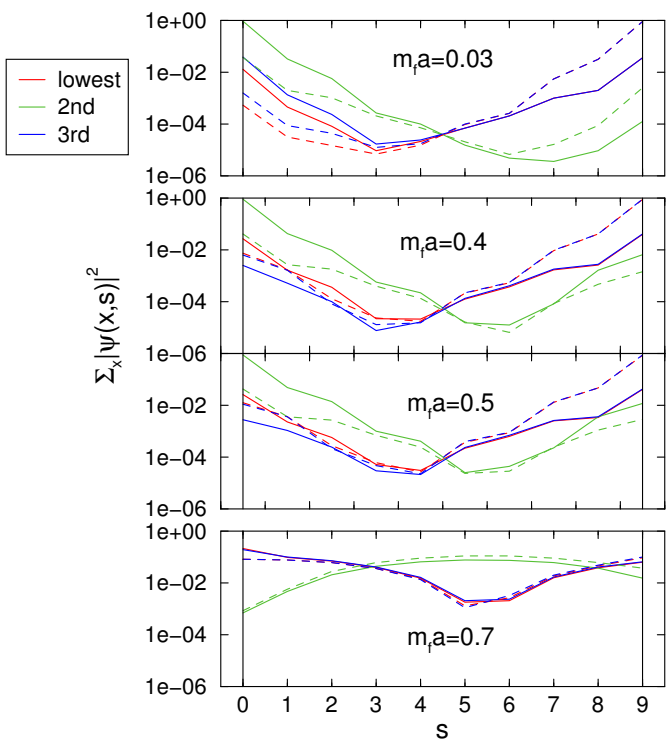

Figure 1: Four-dimensional norms of the low-lying DWF eigenmodes. Courtesy: Jun-Ichi Noaki, RBC Collaboration.

\section{Physics Results}

In Figure 2 we present typical effective mass for the four $J^{P}$ states we are discussing. Similarly reasonable plateaux are obtained for heavy quark masses of $0.1,0.2,0.3$ and 0.4 , and light quark masses of $0.016,0.024,0.032$ and 0.040 . They allow meson mass extraction for all the spin-parity combinations of $J^{P}=0^{\mp}$ and $1^{\mp}$. The pseudoscalar $\left(0^{-}\right)$and vector $\left(1^{-}\right)$mass values are obtained with the light quark mass of 0.008 as well.

Using the known strange mass of $m_{\text {strange }} a=0.0295(14)$ [12], the calculated pseudoscalar heavy light masses with $m_{\text {heavy }} a=0.3$ and 0.4 , and the experimental $D_{s}\left(0^{-}\right)$mass of 1968.3(5)

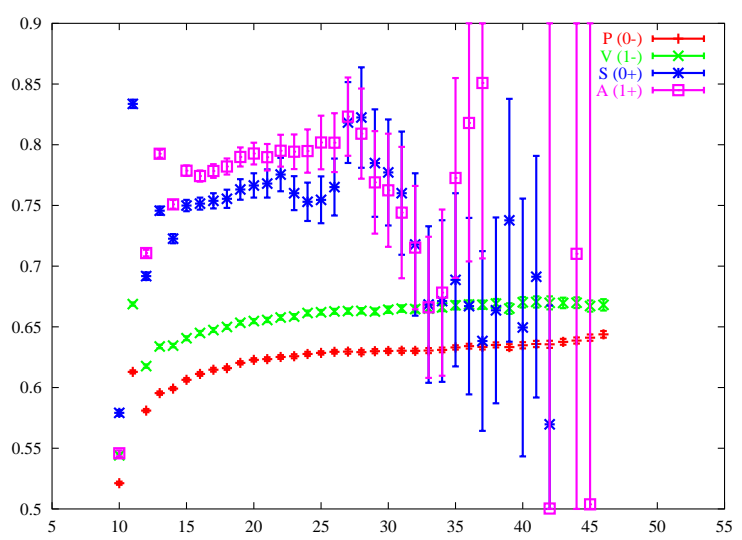

Figure 2: Effective mass of the low-lying heavy light mesons with spin-parity $J^{P}=0^{\mp}$ and $1^{\mp}$, with $m_{\text {heavy }} a=0.3$ and $m_{\text {light }} a=0.040$. 


\begin{tabular}{ll}
\multicolumn{1}{c}{$J^{P}$} & \multicolumn{1}{c}{ this calculation $(\mathrm{MeV})$} \\
\hline \hline$D^{ \pm}\left(0^{-}\right)$ & $1876.7(1.2)(0.4 \%$ over $)$ \\
$D^{* \pm}\left(1^{-}\right)$ & $1968(2)(2 \%$ under $)$ \\
$D_{0}^{*}\left(0^{+}\right)$ & $2362(20)(2.5 \%$ over $)$ \\
$D_{1}^{\prime}\left(1^{+}\right)$ & $2455(17)(1.2 \%$ over $)$
\end{tabular}

\begin{tabular}{ll}
\multicolumn{1}{c}{$J^{P}$} & this calculation $(\mathrm{MeV})$ \\
\hline \hline$D_{s}^{ \pm}\left(0^{-}\right)$ & 1968.3 (input) \\
$D_{s}^{* \pm}\left(1^{-}\right)$ & $2055(6)(2.7 \%$ under $)$ \\
$D_{s 0}^{* \pm}\left(0^{+}\right)$ & $2380(30)(2.5 \%$ over $)$ \\
$D_{s 1}^{* \pm}\left(1^{+}\right)$ & $2460(40)(0.03 \%$ over $)$
\end{tabular}

Table 4: Calculated mass of $D_{s}$ (right, by interpolations for both charm and strange) and $D$ (left, by interpolation for charm and extrapolation for non-strange) states with $m_{\text {charm }}=0.360$ and $m_{\text {strange }}=0.0295$ in lattice units. "over" or "under" refers to the difference from the experimental values. Statistical errors only.

$\mathrm{MeV}$, we set the charm mass as $m_{\text {charm }} a=0.360(7)$ by interpolation. With these values we interpolate to obtain $D_{s}$ states masses and extrapolate to $D$ states ones. The results are summarized in Table 4, and are in reasonable agreement with the experiments, to within a few $\%$.

Parity splittings for the strange $D_{s}$ states turn out as $\Delta_{s, 0}=410(30) \mathrm{MeV}$, about $18 \%$ over estimate compared with $349 \mathrm{MeV}$ experiment, and $\Delta_{s, 1}=380(20) \mathrm{MeV}$, about $10 \%$ over estimate compared with $347 \mathrm{MeV}$ experiment. These calculated values are degenerate within statistical errors. They are also closer to the experiments than previous lattice results. For the non-strange $D$ states they are $\Delta_{s, 0}=487(20) \mathrm{MeV}$, about $11 \%$ over estimate compared with $439 \mathrm{MeV}$ experiment, and $\Delta_{s, 1}=490(17) \mathrm{MeV}$, about $18 \%$ over estimate compared with $417 \mathrm{MeV}$ experiment. Again they are statistically degenerate and are closer to the experiments than the previous lattice results.

In contrast the hyperfine splittings are not so well reproduced: about 87(6) $\mathrm{MeV}$ for $D_{s}$ states, reproducing about $60 \%$ of the $144 \mathrm{MeV}$ experiment, and about 91 (2) $\mathrm{MeV}$ for $D$ states, reproducing about $65 \%$ of the $141 \mathrm{MeV}$ experiment.

The quark mass dependences of parity splittings are summarized in Figure 3. They are statistically indistinguishable for $m_{\text {heavy }} a=0.4$ and 0.3 , but $\Delta_{J=0}>\Delta_{J=1}$ for lighter $m_{\text {heavy }}$ as $\Delta_{J=0}$ increases while $\Delta_{J=1}$ stays more or less constant. The latter behavior may be supported by $K_{1}(1270)-K^{*}(892)$. Both $\Delta_{J=0}$ and $\Delta_{J=1}$ increase toward lighter $m_{\text {light }}$.

\section{Conclusions}

We are studying charm-light meson systems with all the quark flavors described by DWF and with quenched DBW2 gauge ensemble at lattice cutoff of about $a^{-1} \sim 3 \mathrm{GeV}$. Our preliminary conclusions are: DWF well describe charm on the quenched DBW2 ensemble at this cutoff. With interpolations to bare quark mass values of $m_{\text {strange }} a=0.0295$ and $m_{\text {charm }} a=0.360$ in lattice units, the masses of the $J^{P}=0^{ \pm}$and $1^{ \pm} D, D^{*}, D_{s}$ and $D_{S J}$ states are well reproduced to within a few $\%$; their parity splitting, $\Delta_{J}$, are better reproduced than previous works, with only 10-20\% over estimations; the experimental observation of $\Delta_{u d}>\Delta_{s}$ is reproduced; but the hyperfine splittings are only 60-65 \% reproduced. Regarding the dependence on heavy quark mass, $\Delta_{J=0}$ and $\Delta_{J=1}$ are degenerate for $m_{\text {heavy }} a>0.2-0.3 ; \Delta_{J=0}$ increases as $m_{\text {heavy }}$ decreases further while $\Delta_{J=1}$ does not.

We thank RIKEN, Brookhaven National Laboratory and the U.S. Department of Energy for providing the facilities essential for the completion of this work. 

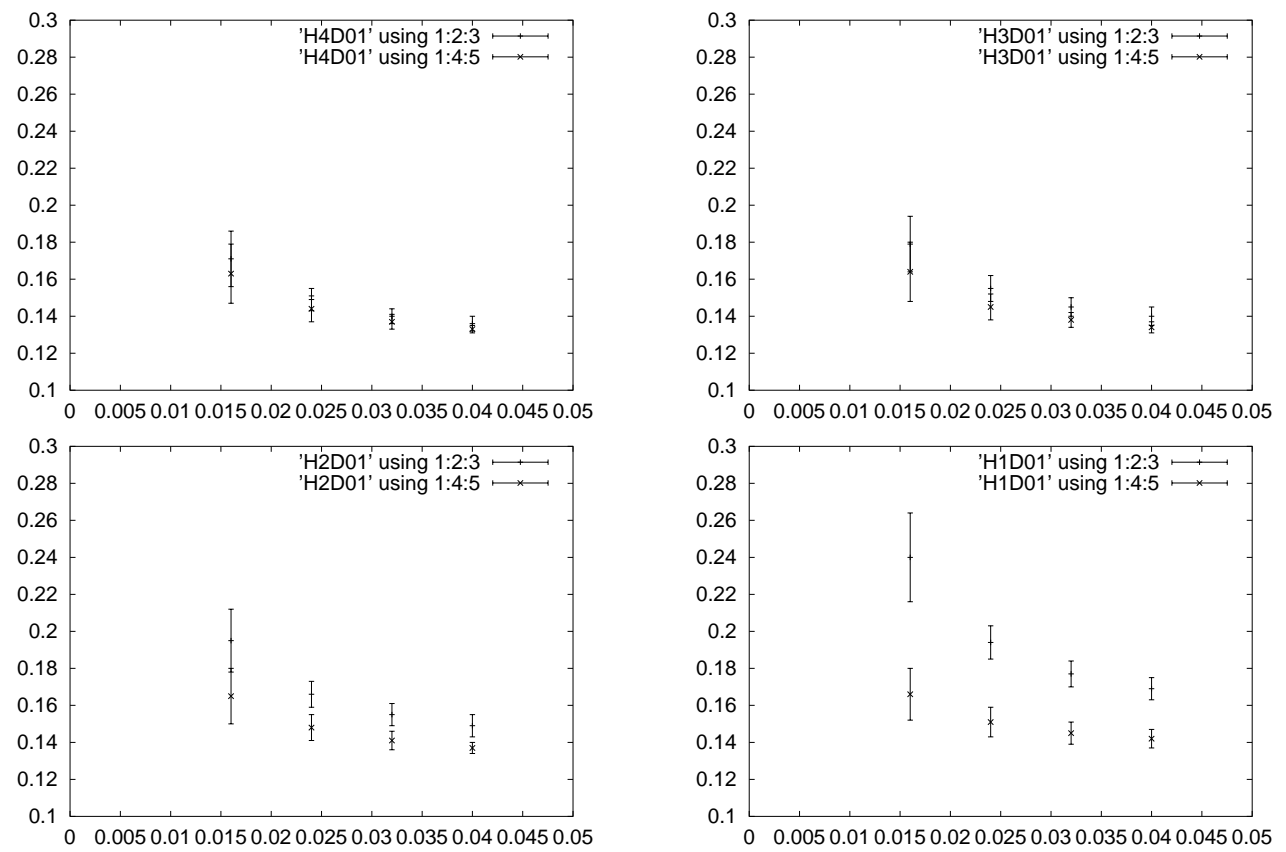

Figure 3: Quark mass dependences of the parity splittings, $\Delta_{J=0}(+)$ and $\Delta_{J=1}(\times)$, with $m_{\text {heavy }} a=0.4$ (upper left panel), 0.3 (upper right), 0.2 (lower left), and 0.1 (lower right). Each panel shows dependence on the light quark mass.

\section{References}

[1] D. B. Kaplan, Phys. Lett. B 288, 342 (1992) [arXiv:hep-lat/9206013];

[2] T. Takaishi, Phys. Rev. D 54, 1050 (1996).

[3] W. A. Bardeen, E. J. Eichten and C. T. Hill, Phys. Rev. D 68, 054024 (2003) [arXiv:hep-ph/0305049].

[4] M. A. Nowak, M. Rho and I. Zahed, Acta Phys. Polon. B 35, 2377 (2004) [arXiv:hep-ph/0307102].

[5] D. Becirevic, S. Fajfer and S. Prelovsek, Phys. Lett. B 599, 55 (2004) [arXiv:hep-ph/0406296].

[6] P. Boyle [UKQCD Collaboration], Nucl. Phys. Proc. Suppl. 63, 314 (1998) [arXiv:hep-lat/9710036].

[7] J. Hein et al., Phys. Rev. D 62, 074503 (2000) [arXiv:hep-ph/0003130].

[8] G. S. Bali, Phys. Rev. D 68, 071501 (2003) [arXiv:hep-ph/0305209].

[9] M. di Pierro et al., Nucl. Phys. Proc. Suppl. 129, 328 (2004) [arXiv:hep-lat/0310045].

[10] A. M. Green, J. Koponen, C. McNeile, C. Michael and G. Thompson [UKQCD], Phys. Rev. D 69, 094505 (2004) [arXiv:hep-lat/0312007].

[11] A. Dougall, R. D. Kenway, C. M. Maynard and C. McNeile [UKQCD], Phys. Lett. B 569, 41 (2003) [arXiv:hep-lat/0307001].

[12] Y. Aoki et al., arXiv:hep-lat/0508011.

[13] K. Hashimoto, T. Izubuchi, and J. Noaki, in these proceedings.

[14] T. Blum et al., Phys. Rev. D 69, 074502 (2004) [arXiv:hep-lat/0007038]. 